\title{
Jóvenes de sectores populares y sentidos sobre la escuela media. La influencia de lo laboral
}

FRANCO CARBONARI

Universidad Nacional de Rosario, Santa Fe, Argentina

\section{RESUMEN}

El presente artículo aborda en clave de exposición los resultados parciales de un estudio cuya problemática central se expresa en la indagación sobre los sentidos que jóvenes de sectores populares construyen en torno a la escuela media, interrogando con mayor detenimiento sobre la incidencia de lo laboral en dicha construcción de sentido. El mismo, centra sus interrogantes en jóvenes cuyo vínculo con la escolarización secundaria y el trabajo ofrece diferentes aristas: que estudian y trabajan, que estudian y no trabajan, que trabajan y no estudian, que no trabajan y no estudian; tanto en instancias formales como no formales. Desde una metodología que articula lo cualitativo y lo cuantitativo, recurrimos a la técnica de la entrevista semiestructurada con la intención de profundizar en el análisis. De esta manera, a partir del relato de los actores, establecemos los vínculos con las categorías de análisis y con datos estadísticos obtenidos de diversas fuentes secundarias.

PALABRAS CLAVE

sentidos; escuela media; jóvenes; trabajo. 


\title{
THE YOUTH FROM LABOR CLASS SECTORS AND THE SIGNIFICANCE OF SECONDARY SCHOOL. THE INFLUENCE OF WORKING
}

\begin{abstract}
This article presents the parcial results of a research which inquires about the significance that the youth from labor class build on secondary school. Special emphasis has been placed on the impact that a working possibility causes at the time of creating a sense of schooling. We focus our concern on teenagers from the labor class since they experience different realities between studying and working. Taking into account both formal and non-formal means there are those ones who do both, while others only study, others work and do not receive education and some neither work nor study. We carried out a semi-structured interview articulating quantitative and qualitative strategies in order to allow deeper analysis. In this way, based on their story, we can establish different links with the categories of analysis and the statistical data obtained from a variety of secondary sources.
\end{abstract}

KEYWORDS

significance; secondary school; youth; work.

\section{JUVENTUDE POPULAR E SENTIDOS EM SETORES E ESCOLA SECUNDÁRIA. A INFLUÊNCIA DO TRABALHO}

RESUMO

Este artigo divulga resultados parciais de um estudo cuja questão central é expressa na pergunta sobre os sentidos que jovens de setores populares constroem em torno da escola secundária, interrogando mais detalhadamente sobre a incidência do trabalho nessa direção. Concentra suas perguntas em jovem cuja ligação com o ensino secundário e o trabalho oferece diferentes aspectos: os que estudam e trabalham, os que estudam e não trabalham, os que trabalham e não estudam, os que não trabalham nem estudam, tanto nas instâncias formais como não formais. Fundamentado em uma metodologia que articula o qualitativo e o quantitativo, recorre-se à técnica de entrevista semiestruturada com a intenção de aprofundar a análise. Assim, com base na história dos atores, são estabelecidas ligações com as categorias de análise e estatísticas derivadas de várias fontes secundárias.

PALAVRAS-CHAVE

sentidos; escola secundária; juventude; trabalho. 


\section{INTRODUCCIÓN}

La pregunta por los sentidos se convirtió en las últimas décadas en un interrogante que no solo tiene a la institución escolar, y sobre todo a la denominada educación secundaria en el centro de la escena, sino que atraviesa todos los estamentos de la vida social y subjetiva. Si bien históricamente, desde su constitución como tal y hasta la actualidad, y desde diversas vertientes político - ideológicas, la pregunta sobre los fines y efectos de la educación ha estado siempre presente, la pregunta por los sentidos presenta una relativa actualidad derivada de mutaciones que exceden a la escuela y que hasta el momento no logran cristalizarse.

A su vez, es innegable el reconocimiento de un fenómeno puntual como incentivo para desandar este camino: el alejamiento de ciertos jóvenes de la escuela media. ${ }^{1}$ Entendemos este fenómeno como uno de los tantos que de manera relacionada desvelan los procesos reflexivos de muchos estudiosos de la presente temática.

Por otra parte, nos hacemos eco de palabras de Duschatzky (1999, p. 18), quien expresará: "Pensar los sentidos sobre la escuela [...] está en la construcción interpretativa que supone inscribir lo dicho en un contexto más amplio de significación". Y en ese sentido es que incluimos en nuestro análisis una variable que históricamente ha dialogado e influenciado en la valoración que una parte de la población ha tenido con relación a la escuela media, dicha variable comprende al mercado laboral.

Por lo tanto, si bien son numerosos los trabajos que analizan los vínculos de los jóvenes con la escolarización secundaria desde la perspectiva de los sujetos en nuestro país (Binstock y Cerruti, 2005; Corea y Lewkowics, 2004; Kantor, 2001; Kessler, 2002; Saintout, 2007), en esta oportunidad, nos urge abordar la problemática acrecentando el énfasis en lo laboral, como elemento que históricamente "ordenó" la configuración de una determinada construcción de sentidos con relación a lo escolar.

Es por esto, $y$ ante la necesidad de actualizar las reflexiones sobre la cuestión planteada, que en el presente artículo nos proponemos acercar significativos avances que se enmarcan en una investigación en curso cuyo interés se centra en explorar, a partir de testimonios de jóvenes de sectores populares de la ciudad de Rosario, los sentidos que estos construyen acerca de la escolarización en el nivel medio, y la incidencia que lo laboral (desde la valoración del "trabajo" hasta la inserción en el mercado laboral) tiene en esa construcción de sentidos. El rastreo se realiza desde tres ejes centrales: La valoración que los jóvenes realizan a cerca de la escolarización en el nivel medio, y los motivos de dicha valoración; el significado que le otorgan a

1 En cantidad de habitantes, la ciudad de Rosario, ubicada en la provincia de Santa Fe, se establece en el tercer lugar detrás de la capital federal y la ciudad de Córdoba. Un dato relevante se da con relación a la temática de la no escolarización de los jóvenes. En clave cuantitativa, las estadísticas censales del año 2010 mostraban que la provincia de Santa Fe se encuentra en el puesto 21 en comparación al resto de las provincias en materia de escolarización de chicos de entre 15 y 17 años, alcanzando esta condición un 76,9\% de esta población específica, lo que habla de un alto porcentaje de jóvenes de esta franja etaria que no estaban escolarizados. Con relación a la ciudad de Rosario, territorio donde se asentará nuestra investigación, los números muestran tendencias similares. 
la cuestión de lo laboral y su percepción de las posibilidades de acceso al mercado de trabajo; la perspectiva y auto proyección con relación al futuro.

Sobre la población con la que se trabaja, creemos oportuno expresar que, si bien la categoría de "joven" conlleva cierta relatividad desde lo etario, por los lazos establecidos con la cuestión laboral (tanto por ciertas urgencias materiales, como así también por mandatos sociales sobre la inclusión del elemento laboral en las proyecciones de vida de los y las jóvenes) trabajamos con jóvenes, hombres y mujeres, que tienen entre 16 y 25 años. $^{2} \mathrm{~A}$ su vez, recuperando los planteos de García Canclini (2004) sobre "lo popular", ${ }^{3}$ el trabajo se lleva a cabo con jóvenes que viven en condiciones signadas por la "subalternidad" material y simbólica con relación a otros sectores sociales.

De esta manera, en un primer momento del artículo damos cuenta del abordaje metodológico de la investigación, posteriormente nos detenemos en la exposición de algunos de los parámetros teóricos desde donde analizamos lo recabado en las instancias de entrevistas. Paso seguido, se procederá a la exposición de dicho análisis. Finalmente damos cuenta de algunas de las conclusiones a las que hemos arribado en la investigación.

\section{MATERIALES Y MÉTODOS}

En primer lugar, cabe mencionar que nuestro objeto de investigación se organiza en torno a una estrategia metodológica de carácter cualitativo y cuantitativo. Es decir, a la pertinente revisión de la amplia bibliografía que existe en torno a

2 Estamos al tanto de que dicha selección comprende solo una parte de los grupos que integran el segmento etario en el que se define la juventud, que en la actualidad comprende entre los 15 y los 29 años de edad CEPAL-OIJ (2004).

3 Para Canclini (2004, p. 164): "Lo popular se constituye como consecuencia de las desigualdades entre capital y trabajo, pero también por la apropiación desigual - en el consumo - del capital cultural de cada sociedad, y por las formas propias con que los sectores subalternos reproducen, transforman y se representan sus condiciones de trabajo y de vida [...]. Lo popular se construye en la totalidad de las relaciones sociales, en la producción material y en la producción de significados, en la organización macroestructural, en los hábitos subjetivos y en las prácticas interpersonales. No pueden ser únicamente las clases, si seguimos entendiéndolas como el marxismo clásico, solo por su posición en las relaciones de producción económica. En la constitución de lo popular también intervienen los aspectos étnicos, sexuales, de consumo, las formas más diversas de lucha contra la represión. Podríamos seguir hablando de clases como sujetos protagónicos si logramos desustancializar su definición e incluir en ella, junto a la participación en el proceso productivo, los demás hábitos, prácticas, creencias, que dan identidad a esos grupos: esas prácticas y esos discursos que se realizan también fuera de la producción, y que a veces no derivan de ella sino de otras determinaciones materiales y culturales. Entre tanto, la noción de popular - cuyas ambigüedades y riesgos se ven claros en los usos racistas, populistas y estatalistas del término - puede servir para identificar la diversidad de relaciones sociales y culturales de los sectores subalternos. Dentro de esta noción amplia, las determinaciones de clase son indispensables para evitar la disolución culturalista de lo popular”. 
nuestro objeto de estudio, tanto estricta como subsidiaria, emprendemos el camino sobre los elementos que constituyen el carácter cuantitativo de la investigación.

Este se encuentra reflejado en la consulta de fuentes secundarias Instituto Nacional de Estadísticas y Censo (INDEC) a partir de la encuesta permanente de hogares $(\mathrm{EPH})$ - , Comisión Económica para América Latina y el Caribe (CEPAL), Dirección Nacional de Información y Evaluación de la Calidad Educativa-Ministerio de Educación Ciencia y Técnica (DiNIECE-MECyT), Sistema de Información de Tendencias Educativas en América Latina (SITEAL), Organización Internacional del Trabajo (OIT) - que expresan aspectos que den cuenta de las condiciones socio económicas que median y potencialmente condicionan los sentidos construidos de jóvenes y adolescentes con relación a la escuela media. A la vez que aportan a esquematizar un acercamiento a la configuración del mercado laboral.

Mientras que, a partir de la realización de entrevistas semiestructuradas, y con el aporte del enfoque antropológico (Achilii, 1987, 2007), ponemos en práctica el aspecto cualitativo de nuestro enfoque metodológico. Con la intención de alcanzar un desmenuzamiento profundo que lleva a la distinción de las reales significaciones, evitando la "fácil generalización", es que abordamos el análisis a partir de dos niveles. En primer lugar, generamos un acercamiento a las interpretaciones que los propios actores tienen de sus acciones y de las problemáticas propuestas en las entrevistas. En segundo lugar, trabajamos con las interpretaciones que permiten los puntos de vista teóricos del investigador sobre las de los actores.

El trabajo de campo se desarrolla en tres barrios de la ciudad de Rosario: Santa Lucía, Puente Negro y Molino Blanco. Si bien con matices, por su constitución socio-laboral (un gran porcentaje de trabajadores con empleos informales, precarios, o sin empleo) y su situación habitacional (casas en muchos casos de chapa habitadas por un gran número de personas), los tres presentan necesidades postergadas, lo que los ubica en cierto lugar de subalternidad.

Hasta el momento llevamos realizadas 19 entrevistas, y con la intención de intensificar la significatividad de los testimonios, se prevé profundizar en algunos casos puntuales.

La selección de los entrevistados incluyó jóvenes mujeres y varones, con un margen etario que va desde los 16 hasta los 25 años, y cuya condición varíe según su situación con relación a aspectos laborales y educativos. Puntualmente, entrevistamos a jóvenes que se encuentran transitando instancias del nivel medio del sistema educativo formal, ${ }^{4}$ como así también no formal, y a la vez trabajan; a su vez que estén atravesando las instancia educativas mencionadas y no trabajan; por otra parte, a jóvenes que se encuentran trabajando pero no han finalizado los estudios del nivel medio y no se encuentran en proceso de finalizarlos, ni participando de instancias educativas no formales; y por último, jóvenes que no se encuentran realizando actividades educativas formales y/o no formales, y tampoco laborales. ${ }^{5}$

4 Inclusive educación para adultos, lo que en la Argentina se denomina Escuela Media para Adultos (EMPA).

5 Cabe aclarar que cuando nos referimos a actividades laborales, lo hacemos con relación a actividades formales como así también informales. 
En este marco, lo que nos proponemos, retomando expresiones de Landreani (1990) es indagar en ese inter-juego entre lo social y lo individual, recuperar ese rasgo característico que en este caso es el sentido interno construido por el sujeto, un sentido cuyo significante está impuesto socialmente, pero cuya imposición no es determinante.

\section{LA CUESTIÓN DEL SENTIDO}

La reflexión sobre la cuestión del sentido es poseedora de unos antecedentes que abarcan campos que alternan entre la sociología, la filosofía, el psicoanálisis, la lingüística, la semiótica, y hasta la hibridación de alguno de estos.

Por ejemplo, desde el campo psicoanalítico, y bajo el sustento de la teoría freudiana del inconsciente, Jacques Lacan, propone pensar el sentido en la órbita de lo Imaginario, y no de lo Simbólico. Esto último, lo Simbólico, es la operación de representación de aquello que llama sentido. Mientras que lo Imaginario, y por lo tanto el sentido, reúne por momentos, todo lo que es ausencia de tiempo, mientras que lo simbólico, por el contrario, suspende esta ausencia, y como ocurrencia lógica abrirá a las funciones inscritas (Lacan, 1974).

Por su parte, desde el campo sociológico, Max Weber establecerá un estrecho vínculo entre el sentido y la acción. De allí que propone la conformación de una sociología de la acción, a diferencia de la sociología a secas. Es que, a partir del análisis de esta, la acción, se establecería el sentido que ordena la misma, o en otras palabras, llevar a su máxima expresión, la lógica que subyace a su sentido"(Marrero, 1999, p. 24). Por su parte, Weber (1992, p. 24) expresará: "En base a los motivos, comprendemos el sentido subjetivo y explicamos el curso de acción".

Por el lado de la filosofía, ante la pregunta por el sentido encontramos en Gilles Deleuze, uno de los fundamentales exponentes de este campo.

Es en el acto de intentar caracterizar la filosofía trascendental que este autor se hará la pregunta por el sentido, y es en el marco de la construcción de este campo trascendental que definirá a aquel como:

[...] la cuarta dimensión de la proposición. Los estoicos la descubrieron con el acontecimiento: el sentido es lo expresado de la proposición, este incorporal en la superficie de las cosas, entidad compleja irreductible, acontecimiento puro que insiste o subsiste en la proposición [...]. Por una parte, no existe fuera de la proposición que lo expresa. Lo expresado no existe fuera de su expresión. Por ello, no puede decirse que el sentido exista, sino solamente que insiste o subsiste. Pero, por otra parte, no se confunde en absoluto con la proposición, tiene una "objetividad" completamente distinta. Lo expresado no se parece en nada a la expresión. El sentido se atribuye, pero no es en modo alguno atributo de la proposición, es atributo de la cosa o del estado de cosas. (Deleuze, 1969, p. 18)

De esta manera, Deleuze sostendrá que el sentido solo podrá manifestarse en la designación de la proposición de un YO que lo porta, que para operar cualquier designación hay que instalarse de golpe en el sentido. 
Por otra parte, instalados en el campo de la filosofía, creemos oportuno rescatar algunos de los planteos realizados por el filósofo contemporáneo Cristobal Holzapfel.

Este distinguirá tres estadios del sentido: existencial, semántico y metafísico. Dirá que el estadio existencial, se encuentra íntimamente ligado a lo que es la justificación de cada acto, de cada decisión que tomamos. Al estadio semántico del sentido lo ubicará en el ámbito del lenguaje, en el que el sentido se presenta relacionado con el significado. Mientras que el metafísico se expresa en términos absolutos, hay sentido o no.

A su vez, la constitución del sentido se produce a partir de una relación entre donación y dotación. Que en todos los fenómenos existe potencialmente una donación de sentido, pero mientras esta no se completa con nuestra dotación, y si se quiere, proyección y construcción, el sentido no se constituye (Holzapfel, 2009).

Uno de los aportes de mayor trascendencia para los fines de nuestra investigación proviene del campo de la semiosis. Desde una perspectiva que retoma elementos del psicoanálisis, Eliseo Verón establecerá un intrínseco vínculo entre los fenómenos sociales y la producción del sentido, entendiendo a esta última como una construcción necesariamente social.

Recuperando la concepción dialógica y contextual de la comprensión, Verón incorpora un esquema desde el cual analizar los sentidos a través del discurso. En el mismo, la comprensión implica responder a un significado mediante otros signos, algo no inmediato y no transparente; confrontación dialógica con otros textos (con-texto) en uno nuevo que articula pasado y futuro (D'Aloisio, 2009).

En palabras de Verón (1987, p. 130):

Una gramática de producción define un campo de efectos de sentido posibles

[...] La red infinita de la semiosis social se desenvuelve en el espacio-tiempo de las materias significantes, de la sociedad y de la historia.

Por su parte, a la lectura contextualizada y dialógica que Verón despliega sobre los sentidos, M. Bajtín incluirá un carácter histórico a la conformación de los mismos. Este autor afirma que los sentidos se renuevan infinitamente en todos los nuevos contextos.

Incluso los sentidos pasados, es decir generados en el diálogo de los siglos anteriores, nunca pueden ser estables (concluidos de una vez para siempre, terminados); siempre van a cambiar renovándose en el proceso del desarrollo posterior del diálogo. (1995, p. 392-393)

\section{EL SENTIDO SOBRE LA ESCUELA MEDIA Y LA INCIDENCIA DE LO LABORAL}

Ahora bien, deteniéndonos momentáneamente en lo concreto, desde una perspectiva socio-histórica, vale expresar que pensar los sentidos construidos alre- 
dedor de la escuela media implica remontarnos a la aparición de dicha institución en nuestro país, y reflexionar sobre los lazos simbólicos establecidos entre ésta y la población. De esta manera, más allá de las profundas modificaciones producidas a lo largo de la historia en el sistema educativo argentino, desde mediados de la década del 40, la denominada escuela media comienza a dar más masivamente asilo a los sectores populares. Con la creación de las escuelas técnicas se empieza a intensificar cierta alianza simbólica de esta con estos sectores de la población, es que veían en dicha institución una promesa de promoción económica y social. De esta manera, y a partir de la mediación del Estado como garante de canalizar estas expectativas ampliando la oferta escolar, ésta, la escuela, potencia su imagen de autoridad indiscutible (Braslavsky, 1985; Tiramonti, 2005).

Por otra parte, desde una perspectiva funcionalista, para reflexionar sobre este lazo simbólico, Francois Dubet (2006, p. 32-61) utilizará la expresión programa institucional, describiendo al mismo:

[...] como el proceso social que transforma valores y principios en acción y en subjetividad por el sesgo de un trabajo profesional específico y organizado [...] Como un tipo de relación social y de trabajo institucional sobre los otros.

Es decir, que la significatividad depositada en la escolarización en el nivel medio excedía la racionalidad instrumental que la ubicaba como instancia previa al acceso a un puesto de trabajo. Sino que la propuesta del programa institucional pudo llevarse a cabo por reposar, como manifiesta el mismo Dubet, sobre una virtud de esperanza.

A su vez, para el sociólogo francés, la caída del programa institucional, o lo que para nuestros fines podríamos traducir como caída de la significatividad que antaño poseía la institución escolar:

[...] proviene de la exacerbación de sus contradicciones latentes, cuando ya no posee la capacidad ideológica para borrarlas [...] la fuerza para superar paradojas por el don de su magia. (Dubet, 2006, p. 64)

Sin embargo, según otro sociólogo francés, Robert Castel (2010), las transformaciones sociales contemporáneas, incluidas las mutaciones del sentido social, se corresponden con las transformaciones que ha experimentado en los últimos 40 años el mundo del trabajo. Puntualmente, sobre estos cambios sociales contemporáneos, incluidos los del mencionado "programa institucional", expresará:

Mi hipótesis es que la onda de choque que nace en el epicentro del trabajo repercute en las diferentes esferas de la existencia social, donde produce efectos diferenciales multiplicados en una formación social que se vuelve cada vez más una "sociedad de los individuos". (Castel, 2010, p. 41)

En términos de lógicas de modelos productivos, que incluye lógicas sobre la organización laboral, esta onda de choque mencionada por Castel implica el pasaje de un modelo de acumulación con asiento predominante en la producción industrial 
a un modelo hegemonizado por el capital de asiento financiero. En palabras de Martín Barbero (2008, p. 39):

De una sociedad industrial, manual, conflictual pero solidaria y negociadora, se comienza a pasar u otra tercerizada, informatizada y menos conflictual pero fracturada, dual, desregulada y excluyente.

Otro de los aspectos que caracteriza estas mutaciones en el "epicentro del trabajo”, según Richard Sennett (2005, p. 18), es el fenómeno de la flexibilidad, sobre dicho fenómeno expresará: "[...] es el nombre que se usa para suavizar la opresión que ejerce el capitalismo".

En otro orden de mutaciones expresadas por el escenario descripto, diferentes autores sostendrán la existencia de procesos de desinstitucionalización, desocialización, despolitización, incluidos el desdibujamiento y la fragmentación de los imaginarios sociales, que afectan particularmente a los jóvenes impulsándolos a un abandono a priori de la ciudadanía (Pérez Sosto y Romero, 2007; Reguillo, 2008; Tenti Fanfani, 2008 apud Tiramonti, 2005).

Ya sea por cuestiones endógenas del propio programa institucional, o por mutaciones en la organización económico - laboral, con sus consecuentes efectos excluyentes sobre una parte de la población, el final del siglo XX, y el comienzo del XXI, expresa una "crisis" de sentidos en la que las instituciones propias de la modernidad, entre las cuales aparece la escuela como estandarte, pierden cierto lugar de "incuestionables".

Por su parte, Duschatzky y Corea (2002) observaban una suerte de pérdida de la autoridad simbólica e impotencia institucional por parte de las instituciones que durante los siglos XIX y XX orientaban el devenir de las personas. Mientras que, sobre el vínculo específico entre escuela y trabajo, el sociólogo argentino Marcelo Urresti (2008, p. 62) va a esbozar:

[...] está aceptado que el conjunto de valores tradicionalmente implícitos que hicieron de la experiencia escolar una preparación para el trabajo, esa cultura común basada en el esfuerzo, el sacrificio y la inversión, pasan por una reestructuración de la que no se sabe todavía sus consecuencias, aunque haya acuerdos sobre el debilitamiento de su validez.

Sin embargo, durante los últimos doce años se llevaron a cabo en Argentina políticas cuyas lógicas no se condecían con las descriptas hasta el momento. Puntualmente,

[...] se registra la configuración de un nuevo régimen de empleo que, junto con el fortalecimiento de las instituciones laborales, generó un marco diferente para las relaciones de trabajo [...]. En este marco se observan el afianzamiento del rol del Estado, un protagonismo recobrado de los sindicatos y una canalización de las demandas salariales a través de la negociación colectiva, fenómenos que contrastan puntualmente con los registrados en la década anterior. Pero también se observan, en contrapunto con aquellas tendencias, la consolidación de tendencias previas, algunas antiguas, otras más recientes. (Palomino, 2010, p. 17) 
En este contexto, algunos de los interrogantes que direccionaron nuestro estudio se podrían ordenar de la siguiente manera: ¿Qué sentidos construyen los jóvenes de sectores populares en torno a la escuela media? ¿Cuál es la incidencia que la configuración del mercado laboral tiene en la conformación de estos sentidos? $\mathrm{Si}$ creemos que el contexto que condicionó estos efectos ha cambiado en pos de mejorar las condiciones y las expectativas laborales ¿¿ué nuevos sentidos se fueron dibujando en torno a la escuela? ¿Esos sentidos han ido cambiando? ¿Cuáles siguen vigentes? ¿Qué grados de sustancialidad engendra dicha reconfiguración?

Por otra parte, si existiera una tendencia general a construir sentidos que valoran positivamente la escuela, ¿Cómo podríamos pensar esta significación que convive con la decisión de los mismos jóvenes de alejarse de la escuela, o de nunca ingresar?

\section{SENTIDOS DE LOS JÓVENES SOBRE LA ESCUELA MEDIA, INCIDENCIA DE LO LABORAL}

Es a partir de la recuperación de expresiones recurrentes de jóvenes entrevistados, como sustento empírico de significatividad para el análisis, en articulación con aportes teóricos sobre la problemática planteada que se presentan las siguientes reflexiones. Es decir, en clave de avances provisorios de nuestra investigación, y como abordaje de los interrogantes recientemente explicitados, en esta instancia ponemos en diálogo aspectos del recorrido desandado con anterioridad en torno a diferentes aproximaciones teóricas sobre la cuestión del sentido, con los testimonios de los propios jóvenes y los pertinentes abordajes teóricos que de estos surgen.

De esta manera, una primera evidencia que emerge del análisis tiene que con la existencia de similitudes y diferencias en las perspectivas según la situación en la que cada joven se encuentra con relación a su vínculo con la escolarización en el nivel medio. Si bien existen aspectos en los que se podrían generalizar perspectivas o sentidos sobre la cuestión escolar, emergen toda otra serie de posiciones diferenciables condicionadas por los trayectos escolares de los protagonistas de dichas trayectorias.

En marco emerge una insoslayable conclusión a partir de los elementos recurrentes en las expresiones de los todos los jóvenes, la misma puede ser explicitada de la siguiente manera:

- Existe una visión estratégica en todos los entrevistados con relación al vínculo escuela media-trabajo. Situación que se relaciona con la percepción de los jóvenes con relación a la obtención de un determinado tipo de empleo. Es decir, la escolarización aparece ligada indefectiblemente a un engranaje según el cual la finalización del nivel medio permitiría alcanzar mejores puestos de trabajo que la no finalización del mismo. Lo que en otros términos se denomina movilidad social ascendente. De esta manera, encontramos en la totalidad de los jóvenes entrevistados una posición según la cual la accesibilidad a un trabajo "estable", "digno", "en blanco", depende de la posesión de la credencial educativa. 
Los siguientes testimonios son una muestra de expresiones que se replicaron en todos los entrevistados:

- Sí, obvio, para conseguir un buen trabajo. Porque yo por ahí la veo a mi mamá que le duele mucho la espalda. Y para trabajar te piden secundaria terminada, qué vos sabés hacer, todo eso te piden.

- ¡Sí! Quiero terminarla para conseguir un trabajo. Ahora cumplo los 18, me anoto en una EEMPA y hago tercero, cuarto, y quinto.

— [...] si yo no llego a terminar la secundaria no sé qué trabajo puedo conseguir.

Mientras que sobre el hecho de la incidencia que tendría la credencial educativa en los tipos de trabajo que se obtengan, rescatamos las siguientes manifestaciones:

- [...] el día de mañana cuando necesiten del estudio se van a dar cuenta ellos solos de que les hacía falta el estudio. Porque no es lo mismo trabajar de limpieza, no saber leer, todas esas cosas: Porque si no sabés leer hoy día no te toman en ningún lado.

- ¿Y qué consejos les darías a esos hermanitos el día de mañana sobre la escuela? - ¡Que estudien! Que estudien todo lo que puedan y que traten de terminar, aunque sea el secundario. Aunque sea eso, ¡porque ya teniendo eso conseguís un trabajo digno!

- ¿Y siempre laburaste en negro?

- Siempre en negro, nunca en blanco. Necesitaría la escuela para tener un buen laburo, un buen trabajo. Si no tenés el secundario completo nadie te llama. Me cansé de tirar currículum.

Demás está decir que la construcción de estas percepciones no está apoyada en estudios sofisticados sobre la incidencia que la escolarización tiene en el tipo de trabajo que se pueda obtener. Si no, que la experiencia de los mismos entrevistados, eso que les ha pasado y no solo lo que ha pasado (Larrosa, 2003), posibilita que las expresiones converjan en esa dirección.

Igualmente, si bien estudios de fines de los años noventa para la zona del Gran Rosario demostraban que la demanda de mano de obra recobraba la particularidad de priorizar sujetos con estudios primarios, más que secundarios, por el tipo de labor requerida (Shapiro, 1997), y otros del nuevo siglo plantean la incapacidad de la credencial educativa del nivel medio de garantizar la movilidad social ascendente (CEPAL, OIJ, 2004; Filmus et al., 2001), la actualidad parece haber quebrado en algún punto esa tendencia. No por una relación directa, descontextualizada y técnica, como en algún punto plantean las teorías del capital humano, sino por definiciones políticas que configuran una determinada lógica del funcionamiento 
económico. ${ }^{6}$ Es ente sentido, podemos afirmar que las percepciones de los jóvenes, apoyadas fundamentalmente en experiencias propias $y /$ o cercanas, se condicen con una tendencia general.

Por otra parte, en consonancia con lo analizado, emerge una constante que tiene que ver con que los jóvenes que no han finalizado su escolarización y no se encuentran transitándola, dan cuenta de una posición de arrepentimiento frente a esa situación. Entendemos dicho emergente como potenciador de los recientes planteos a cerca de la valorización sobre la escuela media.

Pensamos que los siguientes testimonios colaboran con el enriquecimiento de lo enunciado:

- ¿Y cuando dejaste se te cruzó la cuestión del futuro por la cabeza?

- Sííí, porque yo sé que si no tengo estudio no voy a tener trabajo, ni nada. Yo siempre pienso y digo, pero no sé por qué dejé, de loca nomás.

- No me gustaba la escuela, nunca me gustó la escuela. Pero ahora me arrepiento, porque tengo una hija, ¿y qué le voy a decir a mi hija? Porque yo quiero que vaya a la escuela, pero yo no terminé la escuela.

— ¿Y sentís algún tipo de arrepentimiento?

- Y si, obvio, sí sí. Uno, cuando es chico, siempre le dicen "andá, no faltes, pórtate bien", y uno no le da ni pelotas, y cuando después pasa el tiempo, decís: "todo el tiempo que perdí".

6 Un estudio del año 2010 llevado a cabo por Luciana Maresca (Ministerio de Trabajo, 2010, p. 5-7), integrante del equipo de la Encuesta de Indicadores del Mercado de Trabajo (EIMTM), Ministerio de Trabajo, de la Provincia de Buenos Aires, apoyado en la Encuesta Permanente de Hogares (EPH) de la República Argentina, y un informe del Banco Mundial del año 2008, entre otras fuentes, reflejaba lo siguiente: "El período comprendido entre 1998 y 2000 se produce un aumento acelerado en la tasa de desocupación, situándose entorno del 25\%, alcanzando su punto máximo en el año 2002. Este incremento en la desocupación es superior para los grupos con mayor nivel educativo. Esto significa que, durante el transcurso de la crisis, el mercado de trabajo expulsó en mayor medida a los trabajadores con mayor nivel educativo. Se evidencia que la evolución para las personas que poseen estudios universitarios y terciarios fue contraproducente en momento donde se produce la crisis. En dicho contexto el trabajo calificado tiene mayor volatilidad que el trabajo no calificado durante la crisis en el año 2001 [...] el período comprendido entre 1998 y 2000 se produce un aumento acelerado en la tasa de desocupación, situándose entorno del 25\%, alcanzando su punto máximo en el año 2002. Este incremento en la desocupación es superior para los grupos con mayor nivel educativo. Esto significa que, durante el transcurso de la crisis, el mercado de trabajo expulsó en mayor medida a los trabajadores con mayor nivel educativo. Se evidencia que la evolución para las personas que poseen estudios universitarios y terciarios fue contraproducente en momento donde se produce la crisis. En dicho contexto el trabajo calificado tiene mayor volatilidad que el trabajo no calificado durante la crisis en el año 2001". 
Nada más significativo para dar cuenta de un arrepentimiento que la expresión "el tiempo que perdi".Y si uno se pregunta por qué la pérdida de tiempo,por lo intercambiado se desprende que lo que se perdió fue una instancia e incorporación de una herramienta (léase conocimiento o credencial) estratégica para la obtención de un tipo de trabajo.

De esta manera, lo que se infiere, es que, ya sea por la labilidad de algunos diagnósticos, o por una recomposición de los elementos que hacen de la escolarización una herramienta fundamental para el acceso a puestos de trabajo con ciertas características, estaríamos ante la puesta en discusión de las tendencias que auguraban ya sea el desvanecimiento o la disolución de la filiación simbólica entre los sectores populares y la escolarización.

Continuando con los avances que, en clave de conclusiones provisoria, o resultados parciales estamos explicitando, a partir del primero de nuestros arribos, nos vemos inducidos a una segunda afirmación, puntualmente:

- Paralelamente a la perspectiva de los jóvenes acerca de la escuela, explicitada recientemente, se desprende un posicionamiento de estos respecto a la actividad laboral. Es decir, si la escolarización emerge como un medio para alcanzar un "buen trabajo", este último es posicionado como lugar de fin, u objetivo. De esta manera, se evidencia la prioridad significativa de lo laboral al momento de evaluar la importancia de la escolarización. Sin embargo, si nos detenemos en ciertas manifestaciones de dichos jóvenes, encontramos que la finalidad de la obtención de un puesto laboral se relaciona en mayor medida con la intención de obtener una retribución económica, que con aspectos simbólicos que ataño asociaban con mayor intensidad identidades con ocupaciones.

De los testimonios recogidos con anterioridad inferimos lo concluido en esta instancia, a su vez, podemos potenciar dicha inferencia a partir de las siguientes expresiones:

- ¿Y a dónde les gustaría laburar?

-En cualquier lado, con tal de tener plata.

- Haber, imagináte hoy, vos con 22 años, y si no tendrías hijos ¿Estarías buscando trabajo, o te dedicarías a otra cosa?

- Sí estaría buscando. Para tener mis cosas, porque uno piensa en tener sus cosas.

- Yo como recién te dije, yo voy a tener mis cosas, pero las voy a tener por laburar, las voy a tener poniendo el lomo, no necesito salir a hacer nada, yo quiero laburar.

Como se observa, estos testimonios estarían dando cuenta de una situación donde, en términos, socio - históricos, se expresa cierta ruptura con posiciones que reflejaban otro tipo de filiaciones del individuo con lo laboral. Como expresará Florencia Saintuot sobre las representaciones que tiene los jóvenes acerca del trabajo:

El trabajo, para los jóvenes, tiene una dimensión relativa: hoy pueden estar haciendo esto, mañana otra cosa - el trabajo no es un lugar donde quedarse - lo cual erosiona esa dimensión de una identidad sólida. (Saintuot, 2007, p. 111) 
Similar escenario es el que describe el antropólogo argentino Gonzalo Saraví, en un estudio llevado a cabo en el año 2009. A partir del trabajo con jóvenes argentinos y mexicanos arriba a significaciones de parte de estos en las que el dinero emerge como prioridad al momento de otorgar un significado a la tarea laboral. Según su perspectiva:

El trabajo ya no constituye un fin en sí mismo, sino un medio, y su valor radica en esta condición de medio para lograr otros fines. Es en esta esfera, más próxima al consumo que a la producción, en la que el trabajo adquiere relevancia tanto en términos económicos como simbólicos. (Saraví, 2009, p. 56)

En esta instancia, es oportuno recuperar algunas especificidades de nuestra investigación. Puntualmente, si llevamos a cabo el ejercicio de incluir lo dicho por los actores entrevistados en un contexto que se cuela en el texto de las expresiones como sedimentos de un sentido social contemporáneo, se evidencia cierta relación entre unos mandatos ligados fuertemente al consumo (Bauman, 2007), la hegemonía del presente ${ }^{7}$ y el goce inmediato, donde el sacrificio ya no tiene lugar, y las expresiones de los jóvenes. A su vez, no es menor tener en cuenta el sector social del que provienen estos. Ya que, si bien estos bienes simbólicos de época atraviesan a los diferentes grupos sociales, las claves de recepción del mandato en los sectores populares contienen ciertas particularidades. Las mismas pueden relacionarse, en cierta medida con otro de los elementos centrales de nuestra investigación, que es la cuestión de lo laboral. Es decir, si bien como mencionábamos en un comienzo, el presente ofrece ciertas rupturas con la directriz que profundizó la configuración de un mercado laboral caracterizado por lo flexible (en términos de regulación, sobre todo), precario y escaso, la tendencia no se ha disuelto del todo, ni mucho menos, y sobre todo en los jóvenes (Castel, 1995, 2010; Cuervo, 2015; Jacinto, 2010; Miranda, 2008), y sobre todo en los jóvenes de sectores populares.

En este marco, los sentidos construidos sobre lo laboral, que con anterioridad analizábamos, deben inscribirse justamente, en un contexto, donde lo laboral para los jóvenes de sectores populares, en general, es más escaso, precario y flexible que en otro grupo etario. Lo que a su vez puede estar condicionando el vínculo con lo escolar, más allá de las valoraciones que en nuestra primera conclusión remarcábamos. Es decir, de manera dialéctica se produce un vínculo precario entre los jóvenes, sobre todo de sectores populares, con la inserción y desarrollo laboral, influenciado por la nula posesión de credenciales de parte de dichos jóvenes (recordemos que los niveles de no escolarización en el nivel medio son mucho mayores en los sectores de menos recursos que en el resto); situación laboral, que a su vez, puede ser pensada como causa de unos efectos que se expresan en el alejamiento de determinados jóvenes de la intención de obtención de dichas credenciales. Esta compleja situación se potencia al considerar lo que mencionábamos con anterioridad acerca de la existencia de un contexto marcado por una cultura del goce, propugnada por el mercado y los medios de comunicación, donde el sacrificio ya no tiene lugar (Saintout, 2007).

7 "Este mundo del presente está marcado por la ambivalencia de impensado y de lo impensable: impensado del consumo, a imagen de un presente infranqueable caracterizado por la sobreabundancia de los objetos que nos ofrece [...]" (Augé, 2015, p. 32). 
De esta manera, entre estos bienes simbólicos de época, como observábamos, emerge un condicionamiento sobre la construcción de sentido, no solo acerca de la escuela, sino también, del trabajo. En este marco, y como también de manera solapada se ha hecho mención, emerge un elemento relevante como es el de la temporalidad. La temática acerca del tiempo se filtra en muchas de las expresiones de los jóvenes entrevistados, y es parte constitutiva de la construcción de sentidos que estos realizan.

En este marco, el acercamiento al campo nos devuelve diferentes perspectivas con relación al futuro. Como mencionábamos al comienzo del presente apartado, en las entrevistas podemos vislumbrar posiciones comunes, pero también, disonancias acordes a los vínculos establecidos entre los jóvenes y la escolaridad a lo largo de sus trayectorias. A su vez, nos encontramos con casos en que la perspectiva de futuro de jóvenes no escolarizados, pero que tiene algún sostén institucional (iglesia, centro comunitario, trabajo estable) se asemeja a la perspectiva de los jóvenes escolarizados. Por lo tanto, reconocemos en los testimonios puntos comunes acerca de las expectativas futuras, según dicho vínculo.

De esta manera, otro de nuestros resultados parciales es sintetizado del siguiente modo:

- En su gran mayoría, los jóvenes que se encuentran atravesando la escolarización del nivel medio del sistema educativo formal, como así también, aquellos que, si bien no se encuentran escolarizados, sostienen algún tipo de anclaje institucional relacionado con la actividad religiosa, y/o laboral fundamentalmente, ofrecen una perspectiva de futuro menos incierta y precaria que aquellos otros jóvenes desvinculados de los anclajes y prácticas mencionadas. A su vez, la contención de la familia también se inscribe como instancias de gran incidencia en las perspectivas de los jóvenes.

Primeramente, exponemos los aspectos significativos que devuelven las palabras de los jóvenes que sostienen o sostuvieron su escolaridad formal en el nivel medio, o que se encuentran asistiendo a alguna institución que emerge como sostén, o encuentren dicho apoyo en el ámbito familiar. Posteriormente, replicamos los testimonios de aquellos jóvenes que se han desvinculado de la escolarización y de la participación activa en algún otro tipo de institución social.

Entre los jóvenes escolarizados encontramos los siguientes testimonios:

- No, ellos me dicen que por ahora yo siga la escuela, que termine, y que cuando yo sea más grande, que me fije, que, si quiero seguir estudiando, o si quiero trabajar. Pero que lo mejor que hay es terminar la escuela, como ellos no la pudieron terminar, que lo mejor que hay es terminar la escuela para poder ser alguien el día de mañana. — ¿Y ustedes qué sienten? ¿Qué hay oportunidades de hoy día tener un buen futuro? - Sí, yo creo que sí.

- No, yo quiero terminar la secundaria y no quiero estudiar más nada. Quiero pasar los 18, trabajar, y ya hacerme una casa por ahí, no vivir más con mi mamá, nada, tener lo mío, mis cosas, trabajar, depender mí misma. 
- O sea, tu ideal sería tener tu casita, poder mantenerte, con un laburo tranqui y disfrutar...

- Sí, tener lo mío, porque el día que falten mi mamá y mi papá, nosotros no tenemos que depender de esta casa de ellos, tenemos que tener lo nuestro.

- Y a vos dónde te gustaría laburar el día de mañana? ¿Qué laburo te gustaría tener? $\mathrm{O}$ seguir estudiando alguna carrera...

- No, ya... Entrar a una empresa, metalúrgica.

- Che, y de acá a diez años, ¿cómo te imaginas que podés llegar a estar?

- Y, con una casa. Bien. Una familia.

El siguiente testimonio es de un joven que no asistía a un establecimiento educativo, pero se encontraba realizando los trámites pertinentes para retomar sus estudios. A la vez realiza tareas laborales junto a su padre:

- De acá a diez años, ¿cómo te imaginas?

- (Piensa) Te digo la verdad, ni idea. Si te digo te voy a mentir.

- ¿Y para adelante, a dónde apuntás?

- Terminar la escuela para tener un título, porque sin un título no podés hacer nada.

- El título del EMPA. ¿Y después de la escuela? ¿Seguir estudiando, laburando?

- Laburar o estudiar, lo que se me presente.

- ¿Y vos que preferís que se te presente?

- A mi trabajar.

Las expresiones siguientes corresponden a una joven que no ha finalizado la escolarización secundaria, y que tampoco se encuentra realizando tareas laborales (más allá de sus tareas cotidianas como madre), a su vez, en la actualidad participa activamente de la iglesia evangélica:

- ¿Y vos de acá a diez años cómo te ves? Va, si alguna vez te lo pusiste a pensar... - Claro, yo no sé porque yo tuve un hijo, tuve mi marido, tengo mi casa, y de acá a 20 o 30 años no sé lo que me puede pasar también. No sé, yo estoy juntada, yo así me siento bien, todo, me llevo bien con mi pareja. Te digo la verdad, yo con 19 años tengo un hijo, pero yo estoy bien, yo me siento bien estando bien con él. Y es más, veo las cosas que pasan afuera con los jóvenes, ¿`iste? Yo estoy en mi casa, yo voy a la iglesia también, porque yo voy a la iglesia evangélica. Yo estoy ahora en la iglesia, y cambié un montón a como yo era antes.

Como se observa, en alguna medida, los testimonios recogidos muestran una suerte de perspectivas signadas por una relativa certeza de cara al futuro. Situación que nos devuelve varianzas cuando los jóvenes con los que dialogamos no sostienen anclajes institucionales como aquellos sobre los que recuperamos expresiones en primera instancia.

Cuando intercambiábamos sobre aspectos ligados a sus perspectivas de futuro con jóvenes del segundo grupo, esto nos transmitían: 
- Che, y acá a diez años ¿cómo te ves? A los 28.

- Y, a los 28, no sé, capaz que tenga un trabajo, no sé si te digo te miento. Porque falta mucho, no sé si llego.

- ¿Por qué podrías no llegar?

— Y, no sé, de pedo llegué a los 18. Tenía 17 y me dieron 5 tiros, a mi primo le dieron 3 y casi se me murió, al hermano de él.

- Bueno, de acá a cinco años...

- De acá a cinco años capaz que esté trabajando.

Cabe aclarar que estas expresiones pertenecen a Héctor. Un joven de 18 años que a los 8 años debió afrontar el desprendimiento con sus progenitores (su madre presa, y su padre lo abandonó), y a partir de allí su vida transcurre entre casas de parientes y amigos.

A su vez, esto nos decía Miguel:

— Che Miguel, y de acá a un tiempito ¿cómo te ves? A unos diez años más o menos...

— Y, si sigo así no sé si me voy a ver...

- ¿Qué implicaría no verte?

(Silencio casi tenso)

Miguel vive una situación muy similar a la de Héctor. Otro joven nos manifestaba:

— Che y si te ponés a pensar cómo estarías de acá a diez años, ¿cómo te imaginás?

- Para atrás...

- ¿Qué sería para atrás?

- Arruinado, que se yo, mal.

La exposición de abundantes testimonios se inscribe en la necesidad de dar cuenta de una constante que emerge y estaría configurando un mapa de la situación por lo menos preocupante.

$\mathrm{Al}$ igual que en lo concerniente a lo reflexionado sobre las anteriores conclusiones, con relación a la cuestión de las perspectivas de futuro, el análisis amerita el diálogo con una cuestión fundamental, como es el tema de la experiencia. En ese sentido, es que las diferencias que sobre dichas perspectivas expresaron los jóvenes entrevistados podemos inscribirlas en lo experimentado por estos en diferentes tramos de su vida, incluido el presente. Como bien lo señala la socióloga argentina Agustina Corica (2015), recuperando expresiones del historiador alemán Reinhart Koselleck:

En la vida de los jóvenes se ponen en juego experiencias y expectativas que definirán el futuro. Ambas se entrecruzan internamente, no hay expectativas sin experiencias, no hay experiencias sin expectativas. (Koselleck, 1993 apud Corica, 2015, p. 147)

Ahora, bien, por lo observado hasta el momento, con sus señaladas diferencias, es necesario mencionar que las historias de vida de los jóvenes de nuestro estudio, contienen un componente común que es la complicada historia laboral de 
sus progenitores. Aunque cada una con sus particularidades, y si bien en muchos casos muestran inocencia, o desconocimiento, todos los entrevistados al narrar sus historias de vida, reflejan un tipo de vínculo entre sus progenitores y lo laboral atravesado por momentos, por lo menos de inestabilidad.

En este sentido, son muchos los autores que sostienen el intrínseco vínculo entre lo laboral y las expectativas de futuro (Castel, 2010; Corica, 2015; Grinberg y Acosta, 2010; Jacinto, 2010; Saintotut, 2007; Tenti Fanfani, 2008). Lo que constituye para nuestros entrevistados otro elemento experiencial potencialmente condicionante de sus perspectivas. A su vez, como señalábamos con anterioridad, no solo los hechos laborales del pasado, sino que la situación laboral de los jóvenes en el presente crea un posible horizonte de expectativas inciertas.

Retomando a Pierre Bourdieu, Marc Augé expresará:

Si el tiempo parece aniquilarse, es porque el trabajo es el soporte, si no el principio, de la mayoría de los intereses, expectativas, exigencia, esperanzas e inversiones en el presente ( $y$ en el futuro o el pasado que este implica), en suma, uno de los fundamentos mayores de la ilusio como compromiso en el juego de la vida, en el presente, como presencia en el juego, por lo tanto en el presente y el futuro, como inversión primordial que - todas las sabidurías lo enseñaron siempre identificando el desarraigo del tiempo al desarraigo del mundo - hace el tiempo, es el tiempo mismo. (Augé, 2015, p. 95)

$\mathrm{Si}$, como señala la OIT en un estudio sobre el empleo para los jóvenes, ${ }^{8}$ se efectuaron ciertos avances en el contexto latinoamericano, pero este sigue siendo un bien escaso para esta población, entonces estaríamos frente a un elemento que se inscribe en el contexto en el que esta construye sentidos. $E$ indefectiblemente, una "lábil" perspectiva de futuro incidirá en un lábil vínculo con una institución

8 Luego de la contracción económica de 2008-2009, América Latina y el Caribe experimentaron una recuperación que se vio reflejada en los indicadores del empleo. El desempleo urbano en 2011 se situó en un nivel del 6,8\%, nivel que no se veía desde los años de 1990. A su vez mejoraron otras condiciones relacionadas con el empleo y el trabajo decente, como el acceso a la seguridad social, a mejoras en los salarios mínimos reales y en las remuneraciones medias. Sin embargo, si bien el desempleo ha bajado, la proporción de trabajadores por cuenta propia y auxiliar en actividades de baja productividad sigue alta, cerca de un tercio del total del empleo en la región. Por otra parte, un $44 \%$ de los trabajadores y trabajadoras aún no tienen ningún tipo de cobertura. A su vez, los jóvenes registran un porcentaje de desocupación que triplica al de los adultos (14,9\% para los jóvenes y 5\% para los adultos). Seis de cada diez que consiguen trabajo solo tienen acceso a empleos informales, en condiciones de precariedad laboral, desprotección social y con baja productividad e ingresos. De aquellos que logran empleo en el sector formal de empresas, 37 de cada 100 no están protegidos por la seguridad social, porque en la práctica acceden a empleos precarios y son más vulnerables a los ciclos económicos. Si bien es del año 2011, las tendencias del informe siguen presentes. (Organización Internacional del Trabajo, Panorama Laboral 2011, OIT, Oficina Regional para América Latina y el Caribe, 2011). 
que se vuelve estratégica en la elaboración de un proyecto a largo plazo como es la escuela. En otros términos:

[...] en una sociedad en la que ya no puede establecerse una relación lineal entre educación y ocupación, la justificación de la enseñanza en su utilidad ya no se hace posible. (Grinberg y Acosta, 2010, p. 45)

Todo parecería indicar, a partir de lo expresado en este último tramo, que ciertos hechos de la estructura económica que afectan experiencialmente a los implicados en nuestro análisis estarían incidiendo en un posicionamiento más bien precario con relación a sus perspectivas de futuro. En este sentido, emergen otro tipo de planteos que corren al agente del lugar de la pasividad y el perjuicio y esbozan la idea de que cierta ruptura con la vivencia lineal del tiempo podría implicar de manera indirecta una suerte de libración de una lógica que determinaba con algunos niveles de inexorabilidad esa vivencia. Si bien el hecho es pensado como efecto de transformaciones estructurales perjudiciales para ciertos sectores sociales desde el punto de vista económico, esto posibilita justamente poner en discusión qué implica una organización social donde el tiempo sea apropiado desde una lógica instrumental, donde lo útil prevalezca sobre el instante. Es decir, si los jóvenes se ven reactivos ante la propuesta de elaborar una proyección de sus vidas, y en esto influye lo fluctuante de los horizontes, aspecto que a su vez impacta en la escolarización de estos, también emerge la posibilidad de preguntarnos qué posibilidad de ofertas se abre desde el punto de vista pedagógico para la construcción de nuevos sentidos para lo escolar, y en definitiva, para lo temporal, o qué tiene para proponer la escuela cuando cae la función instrumental por la ausencia del objetivo de dicho instrumento.

Retomando lo específico de nuestro estudio, cabe recordar que tampoco sería correcto homologar todas las posiciones de los entrevistados a situaciones particulares. Ya que como hemos observado, aquellos jóvenes entrevistados que cuentan con unos anclajes en determinados espacios de contención, ya sea institucional y/o familiar, mantienen ciertos cimientos que inciden en cómo se posicionan de cara al futuro, a diferencia de aquellos que no.

Finalmente, no queríamos dejar de hacer mención de una constante en la opinión de los jóvenes. Ya que en nuestro trabajo de campo fueron recurrentes expresiones en las que subyacían principios del orden del sentido común que en las representaciones de la sociedad circulan sin mayor criterio de criticidad. Las mismas tienen que ver con la absoluta autorresponsabilidad del vínculo nulo, de baja intensidad (Kessler, 2005), o vulnerabilidad sostenido por algunos jóvenes con la escolarización.

Estos principios se hicieron presentes en la casi totalidad de los entrevistados. Cuando se los consultó sobre los motivos por los cuales creían que los jóvenes dejan la escuela, las respuestas giraban en torno a ideas tales como: "Mirá, cada uno hace lo que quiere. También hay que tienen problemas"; "Porque se quieren quedar en la esquina drogándose, quieren que la mamá los mantenga, o el papá"; "No sé por qué no van. Capaz que se aburren, o... Siempre pasa algo, o problemas familiares, o 
se aburren de la escuela, o no les gusta la escuela"; "No, no sé. Les gusta la calle, la droga, todo. Por eso no van. Ya ni los padres manejan a los pibes".

Como se evidencia, la situación en ningún momento es pensada en términos de problemática social, ya sea por decisión individual o problemas del ámbito doméstico, la configuración social (dígase desigualdades), y/o pedagógica (la escuela es aburrida, y no los jóvenes se aburren), quedan perimidas en los discursos de estos jóvenes.

\section{A MODO DE CIERRE}

Como se señalaba al inicio del presente artículo, la reflexión sobre los sentidos se convierte en un recurrente ejercicio de la contemporaneidad. Si bien desde diversos campos teóricos ha sido, y es, un elemento que en tanto objeto de la curiosidad epistémica se hace presente en diferentes propuestas analíticas, a partir de algunos sucesos significativos (léase mutaciones en los patrones de acumulación económica con su consecuente influencia sobre la organización del campo productivo-laboral, o declive del "programa institucional") la actualidad refleja un manto de incertidumbres que exigen del ámbito académico un esfuerzo por amainar las dudas.

De esta manera, con relación a los interrogantes que atañen a nuestro estudio, lo expuesto pretende dar cuenta de una compleja trama en la que lo relatado por jóvenes que viven en barrios de sectores populares de la ciudad de Rosario emerge como referencia.

En ese sentido se evidencia una fuerte y recurrente valoración sobre la obtención de una credencial del nivel medio, lo que daría cuenta de un sentido instrumental con relación a la escolarización. Ya sea por experiencias propias, o cercanas, o por discursos de los cuales se apropian estos jóvenes, la idea de que la finalización del nivel medio se constituye en garantía de trabajo relativamente, y por lo menos, más estable, que la no obtención de la credencial, es constante. Lo que expresaría la existencia de una percepción en la que la movilidad social ascendente se hace presente.

A su vez, como mencionábamos, si la escolarización emerge como un medio para alcanzar un "buen trabajo", este último es posicionado como lugar de fin, u objetivo. De esta manera, lo laboral emergería como una significativa prioridad al momento de evaluar la importancia de la escolarización. Sin embargo, la obtención de un puesto laboral se relaciona en mayor medida con la intención de obtener una retribución económica, que con aspectos simbólicos que ataño asociaban con mayor intensidad identidades con ocupaciones.

Otros de los aspectos condicionantes del tipo de filiación que los sujetos puedan construir con lo escolar, se refleja en las perspectivas de futuro que estos sostengan. A su vez, como observábamos, dicha perspectiva está supeditada, en primer lugar, a los niveles de escolarización alcanzados, pero a la vez, a elementos experienciales sobre la propia historia familiar y su vínculo con lo laboral, como así también, a ciertos anclajes en instituciones sociales o del plano familiar, que éstos mantengan. Aspectos, éstos que podrían orientarnos en el entendimiento sobre la contradicción entre la importancia que es otorgada a la escuela como 
instancia estratégica para la obtención de un trabajo, y en muchos casos, su no concurrencia a dicha institución.

En este sentido, emerge una operación muy riesgosa, la que, como hemos observado, responsabiliza en primer lugar a los propios sujetos que vivencian esta situación de "des-institucionalización”, y, en segundo término, las vincula a problemáticas del ámbito doméstico. Dicha operación podría estar incidiendo en una autopercepción, o en una construcción de sí mismos en la que la idea de la incapacidad, y/o imposibilidad opera inhibiendo.

Una de las instancias en que esto se hace presente tiene que ver con la inacción de los jóvenes para retomar sus estudios, a pesar de estar en condiciones formales, y sentirse arrepentidos por su inconclusa escolarización en el nivel medio del sistema educativo formal.

En este marco, la pregunta por los sentidos dispara hacia las direcciones menos esperadas. Por eso, la necesidad de insistir, enfatizando en la mirada de los jóvenes, pero de manera relacionada en los intersticios institucionales y sociales que hacen de afluentes experienciales a partir de los cuales estos construyen dichos sentidos.

\section{REFERENCIAS}

Achilli, E. Notas para una antropología de la vida cotidiana. Cuadernos de la Escuela de Antropología, Rosario: Facultad de Humanidades y Artes, v. 24, n. 2, p. 81-93, 1987. . Investigación educativa y escuela pública: algunas perspectivas y debates. JoRnAdAs de Investigación en EduCACión. Educación y perspectivas: contribuciones teóricas y metodológicas en debate, 5., 2007, Córdoba. Anais... Córdoba: Centro de Investigaciones "María Saleme de Burnichon" y Escuela de Ciencias de la Educación; Facultad de Filosofía y Humanidades; Universidad Nacional de Córdoba, 1-2 jul. 2007. p. 29-38.

Augé, M. ¿Quépasócon la confianza en el futuro? Buenos Aires: Siglo XXI Editores, 2015. Bajtín, M. Estética de la creación verbal. México: Siglo XXI, 1995.

Barbero, J. M. Reconfiguraciones de la comunicación entre escuela y sociedad. In: Tenti Fanfani, E. (Comp.). Nuevos temas en la agenda de politica educativa. Buenos Aires: Siglo XXI, 2008. p. 65-100.

Bauman, Z. Vida de consumo. Buenos Aires: Fondo de Cultura Económica, 2007.

Binstock, G.; Cerruti, M. Carreras truncadas: el abandono escolar en el nivel medio en la Argentina. Buenos Aires: UNICEF, 2005.

Braslavs ky, C. La discriminación educativa en Argentina. Buenos Aires: Miño y Dávila Editores, 1985.

Castel, R. La metamorfosis de la cuestión social: una crónica del salariado. Buenos Aires: Paidós, 1995.

. El ascenso de las incertidumbres. Trabajo, protecciones, estatuto del individuo.

Buenos Aires: Fondo de Cultura Económica, 2010. 
CEPAL — Comisión Económica para América Latina y el Caribe. OIJ — Organismo Internacional de Juventud para Iberoamérica. Panorama social de América Latina. Santiago de Chile: 2004. Disponible en: <http://repositorio.cepal.org/bitstream/ handle/11362/1221/S0480609_es.pdf;jsessionid=3DEC83DDFC273D0554A434F 4C2CCBAE0?sequence=1>. Acceso en: ago. 2015.

Corea, C.; Lewrowics, I. Pedagogía del aburrido: escuelas destituidas, familias perplejas. Buenos Aires: Paidós, 2004.

Corica, A. Juventud y futuro. In: Miranda, A. (Org.). Sociología de la educación y transición al mundo del trabajo. Buenos Aires: Teseo, 2015. p. 145-168.

Cuervo, H. Apuntes para una teoría de la justicia plural en los estudios de la juventud. In: Miranda, A. Sociología de la educación y transición al mundo del trabajo. Buenos Aires: Teseo, 2015. p. 53-76.

D'Aloisio, F. Aportes de las teorías hermenéutica y semiótica en la investigación sobre sentidos sociales. Córdoba: UNC-CONICET, 2009. Disponible en: <http://sedici.unlp. edu.ar/bitstream/handle/10915/17325/Documento_completo__.pdf?sequence=1 >. Acceso en: oct. 2013.

Deleuze, G. Lógica del sentido. Edición electrónica de www.philosophia.cl/Escuela de Filosofía. Universidad ARCIS. Traducción Manuel Morey.[S.1.: s.n.], 1969. Disponible en: <http://www.uruguaypiensa.org.uy/imgnoticias/588.pdf>. Acceso en: 21 feb. 2018.

Dubet, F. El declive de la institución: profesiones, sujetos e individuos en la modernidad. Buenos Aires: Gedisa, 2006.

Duschatzky, S. La escuela como frontera. Reflexiones sobre la experiencia escolar de jóvenes de sectores populares. Buenos Aires: Paidós, 1999.

Duschatzky, S.; Corea, C. Chicos en banda. Los caminos de la subjetividad en el declive de las instituciones. Buenos Aires: Paidós, 2002.

Filmus, D.; Kaplan, C.; Miranda, A.; Moragues, M. Cada vez más necesaria, cada vez más insuficiente: escuela media y mercado de trabajo en épocas de globalización. Buenos Aires: Academia Nacional de Educación; Santillana, 2001.

García Canclini, N. ¿De qué hablamos cuando hablamos de lo popular? Culturas populares e indígenas. Revista Diálogos en la Acción, primera etapa, México: Dirección General de Culturas Populares e Indígenas, p. 153-165, 2004. Disponible en: <http:// hugoribeiro.com.br/biblioteca-digital/Canclini-de_que_estamos_hablando_cuando_ hablamos_de_lo_popular.pdf>. Acceso en: jul. 2015.

Grinberg, S.; Acosta, F. Pedagogía y subjetividad. In: Donini, A. M. (Coord.). Nuevas infancias y juventudes. Una experiencia formativa. San Martín: UNSAM Edita, 2010. p. 29-52.

.Diálogos en la revista con Cristóbal Holzapfel. Revista Medicina y Humanidades, Santiago de Chile: Campus Sur de la Facultad de Medicina de la Universidad de Chile, v. I, n. 3, p. 131-149, sept./dic. 2009. Disponible en: <http://www.medicinayhumanidades. cl/ediciones/2009/numero_3/13_Entrevista.pdf >. Acceso en: nov. 2011. 
Jacinto, C. (Comp.). La construcción social de las trayectorias laborales de jóvenes políticas, instituciones, dispositivos y subjetividades. Buenos Aires: Teseo/IDES; Programa de Estudios sobre Juventud, Educación y Trabajo (PREJET-IDES), 2010.

Kantor, D. (Coord.). La escuela secundaria desde la perspectiva de los jóvenes con trayectorias escolares inconclusas. Informe final. Buenos Aires: Dirección General de Planeamiento; Secretaría de Educación; GCBA, 2001.

Kessler, G. La experiencia escolar fragmentada: estudiantes y docentes en la escuela media en Buenos Aires. Buenos Aires: Instituto Internacional de Planeamiento de la Educación; IIPE; UNESCO; Sede Regional, 2002.

. Sociología del delito amateur. Buenos Aires: Paidós Ibérica, 2005.

Lacan, J. Seminario 22: R.S.I. — versión crítica - Clase 1. [S.1.: s.n.]: 10 dic. 1974. Disponible en: <http://www.lacanterafreudiana.com.ar/2.1.10.1\%20CLASE\%20 -01\%20\%20S22.pdf>. Acceso en: enero 2016.

Landreani, N. Métodos cuantitativos versus métodos cualitativos: un falso dilema. Revista Ciencia, Docencia y Tecnología, Entre Ríos: UNER, n. 1, año I, p. 1-28, mar. 1990. Larrosa, J. La experiencia de la lectura. Estudios sobre literatura y formación. México: Fondo de Cultura Económica, 2003.

Marrero, A. La sociología explicativo-comprensivista de Max Weber un reexamen. Documentos de Trabajo, Montevidéu: Universidad de la República, n. 48, p. 1-39, 1999.

Ministerio de Trabajo. EIMTM - Encuesta de Indicadores del Mercado de Trabajo en los Municipios de la Provincia de Buenos Aires. Municipio de Cañuela, Argentina, 2010. Disponible en: <http://www.trabajo.gba.gov.ar/informacion/pdf_eimtm/2010/ INFORME_CANUELAS_2010.pdf>. Acceso en: nov. 2016.

Miranda, A. Los jóvenes, la educación secundaria y el empleo a principios del siglo XXI. Revista de Trabajo, Buenos Aires: Ministerio de Trabajo, Empleo y Seguridad Social de la República Argentina, Subsecretaría de Programación Técnica y Estudios Laborales, año 4, n. 6, p. 185-198, ago./dic. 2008.

Palomino, H. Las nuevas dinámicas de las relaciones laborales en Argentina. Buenos Aires: Jorge Baudino Ediciones, 2010.

Pérez Sosto, G.; Romero, M. La cuestión social de los jóvenes. 2007. Artículo basado en el esquema conceptual de la etapa diagnóstica y propuestas del estudio "Trabajo decente para los jóvenes en Argentina”. Argentina: OIT, 2007. Disponible en: <http:// www.fundacion.uocra.org/documentos/recursos/articulos/La-cuestion-social-de-losjovenes.pdf>. Acceso en: jun. 2011.

Reguillo, R. Instituciones desafiadas. Subjetividades juveniles: territorios en reconfiguración. In: Tenti Fanfani, E. (Comp.). Nuevos temas en la agenda de política educativa. Buenos Aires: Siglo XXI, 2008. p. 125-144.

Saintuot, F. Jóvenes e incertidumbre. Percepciones de un tiempo de cambio: familia, escuela, trabajo y política. 2007.310f. Tesis (Doctorado en Ciencias Sociales) — Facultad Latinoamericana de Ciencias Sociales Sede Académica Argentina, Buenos Aires, 2007. 
SARAví, G. Juventud y sentidos de pertenencia en América Latina: causas y riesgos de la fragmentación social. Revista CEPAL, México: CEPAL, n. 98, p. 47-65, ago. 2009.

Sennett, R. La corrosión del carácter. Consecuencias personales del trabajo en el nuevo capitalismo. La Habana: Editorial de Ciencias Sociales, 2005.

Shapiro, J. El empleo en el aglomerado Gran Rosario 1994/1996. Cuadernos del Gran Rosario, Rosario: [s.n], p. 70-74.

Tenti Fanfani, E. (Comp.). Nuevos temas en la agenda de politica educativa. Buenos Aires: Siglo XXI, 2008.

Tiramonti, G.(Comp.). La trama de la desigualdad educativa. Mutaciones recientes en la escuela media. Buenos Aires: Manantial, 2005.

Urresti, M. Nuevos procesos culturales, subjetividades adolescentes emergentes y experiencia escolar. In: Tenti FAnfani, E. (Comp.). Nuevos temas en la agenda de politica educativa. Buenos Aires: Siglo XXI, 2008. p. 53-64.

Verón, E. La semiosis social. Buenos Aires: Gedisa, 1987.

Weber, M. Economía y sociedad. México, DF: Fondo de Cultura Económica, 1992.

\section{SOBRE EL AUTOR}

Franco Carbonari es doctor en educación por la Universidad Nacional de Entre Ríos (Argentina). Profesor de la Universidad Nacional de Rosario (Argentina). E-mail: orko215@hotmail.com

Recibido el 26 de enero de 2016

Aprobado el 4 de abril de 2016 